Research Article

\title{
Investigating Driving Styles: A Validation Study of Multidimensional Driving Styles with British and Chinese Drivers
}

\author{
Xu Sun, ${ }^{1}$ Ying Jiang, ${ }^{1}$ Gary Burnett, ${ }^{2}$ and Qingfeng Wang ${ }^{3}{ }^{3}$ \\ ${ }^{1}$ Faculty of Science and Engineering, University of Nottingham Ningbo, Ningbo 315100, China \\ ${ }^{2}$ Human Factors Research Group, University of Nottingham, Nottingham, UK \\ ${ }^{3}$ Nottingham University Business School China, University of Nottingham Ningbo, Ningbo 315100, China \\ Correspondence should be addressed to Qingfeng Wang; qingfeng.wang@nottingham.edu.cn
}

Received 29 September 2020; Revised 26 August 2021; Accepted 19 September 2021; Published 30 September 2021

Academic Editor: Nicola Baldo

Copyright (C) $2021 \mathrm{Xu}$ Sun et al. This is an open access article distributed under the Creative Commons Attribution License, which permits unrestricted use, distribution, and reproduction in any medium, provided the original work is properly cited.

\begin{abstract}
Culture has a significant impact on driving behaviour and can play an important role in driving safety. The adaptation of trafficrelated psychological instruments, developed elsewhere in new national contexts, should consider the cultural context. This paper validates the multidimensional driving style inventory (MDSI) with two cultural samples consisting of 215 Chinese drivers and 240 British drivers. A factor analysis of the driving style yielded evidence that both datasets present some variations from the original version of the instruments in the factorial structure. The analysis of the UK sample is comparable to the previous MDSI by indicating six driving styles, namely, anxious, risky and dissociative, high-velocity and angry, patient, careful, and distressreduction. The analysis of the Chinese participants' dataset showed its factorial structure with 40 items of the 44 original items divided over six styles. A new dimension, namely, an inattentive driving style, appeared in the Chinese sample. These differences raise the need to validate and adapt such instruments to consider cultural specificities. Implications were also derived for driver and road safety enhancement solutions through driver behaviour applications.
\end{abstract}

\section{Introduction}

Road traffic accidents are a major health and economic problem throughout the world, and research within traffic psychology has mainly focused on the human factors that are evident in car accidents, such as attitudes, driving behaviours, and sociodemographics (e.g., [1, 2]). Driving styles have been shown to be an important human factor related to traffic accidents [1]. Rothengatter and Huguenin [2] have suggested that $85 \%$ of traffic accidents can be attributed to human factors, but it remains necessary to explore this issue more deeply and broadly. Elander et al. [3] defined driving style as the method drivers choose to employ when they are driving, or how they drive habitually while facing various traffic conditions, including their choice of driving speed, the distance from vehicles ahead, and their habitual level of general attentiveness and assertiveness. It has been shown that drivers with certain driving styles, such as being reckless and careless, tend to exhibit unsafe driving behaviours [4].
Therefore, an investigation of driving styles can help us to better understand the causes of certain kinds of driving behaviour from a more general perspective. It can also help to provide important support for the classification of drivers, such that drivers with different driving styles could be monitored and alerted in advanced driver-assistance systems to reduce risky driving behaviours.

To identify people's driving styles, two approaches have been widely applied, including simulator experiments and self-reported surveys. Simulator experiments have the advantage of allowing researchers to establish customized environmental conditions; however, such experiments are usually expensive and are limited by the reality of a virtual environment [5]. Therefore, self-reported surveys have become a rather popular approach. The multidimensional driving style inventory (MDSI) is a self-report measure developed to assess driving styles [6]. A total of 44 items were developed by Taubman-Ben-Ari et al. [7] to measure driving styles according to a subjective approach, in which 
participants were asked to rate, on a 6-point scale, the extent to which each item reflected their feelings, thoughts, and behaviours as drivers. The factor analysis revealed eight main driving style factors, which explained $56 \%$ of the variance of the 44 items, namely, dissociative, anxious, risky, angry, high-velocity, distress-reduction, patient, and careful. Based on an extensive literature review, Taubman-Ben-Ari et al. hypothesized four broad dimensions of driving styles. The first dimension is characterized by driving at high speed, passing other cars in no-passing zones, and driving while intoxicated, probably endangering both the driver and others. This dimension is represented in the MDSI by the risky and high-velocity driving styles. The second dimension is where drivers exhibit feelings of alertness and tension. This dimension is represented by anxious, dissociative, and distress-reduction styles. The angry driving style is the third general dimension, reflecting a tendency to drive aggressively on the road, such as sounding the horn or flashing lights at other drivers. Finally, there is the patient and careful driving style, in which drivers are well-adjusted, patient, and polite while following the rules. This is represented in the MDSI by the careful patient style.

Taubman-Ben-Ari et al. [7] have provided evidence of the reliability and validity of the MDSI as applied to the population of Israel. Furthermore, the MDSI has already been translated into several languages and has been verified in a variety of countries (e.g., Argentina, Belgium, the Netherlands, Spain, Romania, Malaysia, and Italy). Poó et al. [8] adapted and validated a Spanish version of the MDSI for use in Argentina, reducing the dimensions to six factors, by merging high velocity with risky, and patient with care. Trógolo et al. [9] also examined the validity of the MDSI with a sample of 1277 drivers from Argentina. Exploratory structural equation modelling (ESEM) was used to test the factor structure and measurement invariance of the MDSI. The findings suggested a 6-factor model represented by the risky, angry, dissociative, anxious, careful, and patient styles. Huysduynen et al. [10] further validated the MDSI for drivers in Belgium and the Netherlands. Their study found that the five factors identified among participants from the Netherlands and Belgium were more stable than the eight factors from the original study with the participants in Israel. The following five factors were identified: angry, anxious, dissociative, distress-reduction, and careful. Padilla et al. [11] validated the appropriateness of the MDSI to the Spanish spoken in Spain. The results revealed a 6-factor structure of the Argentinian version of the MDSI, with higher internal consistency values for each of the driving styles. Karjanto et al. [12] investigated the driving styles using the MDSI with 338 drivers in Malaysia. The findings revealed four driving styles out of the original eight factors, including careful, risky, anxious, dissociative, and angry styles. Holman and Havârneanu [13] developed a Romanian version of the MDSI to fit the Romanian driving context and cultural specificities. It divided driving behaviour into seven styles, among which the anxious, risky, angry, distress-reduction, and dissociative styles correspond to the original instrument. A supplementary driving style, namely the "violation of rules", contextually perceived as the irrational style, was introduced. Freuli et al. [14] validated the MDSI for the measurement of driving styles with 561 drivers from Italy. They confirmed the 8-factor structure of the original MDSI by removing the 4 items which did not properly contribute to the factors. Wang et al. [15] examined the reliability, structure, and validity of a Chinese version of the MDSI with 296 drivers from China, to determine which types of driving styles are typical among Chinese drivers. They revised the original MDSI by indicating four driving styles, namely, the risky, angry, highvelocity, careful, and anxious styles. Long and Ruosong [16] also validated a Chinese version of the MDSI with 760 drivers. Their study revealed a 6-factor structure of the MDSI including risky, anxious, angry, distress-reduction, careful, and dissociative driving styles. These studies have all supported the reliability and validity of the MDSI; however, the factor structure encountered across the studies has been inconsistent.

There is evidence that cultural factors influence both traffic performance and safety [17]. For example, Chinese drivers have a unique traffic culture that could promote different driving styles and behaviours $[18,19]$. In studies concerned with displays of anger while driving, Chinese drivers reported less anger in response to discourtesy and illegal driving than their American counterparts, or drivers in New Zealand and Spain [20]. In terms of driving skills, Chinese drivers exhibit a different driving style and are involved in more crashes than those in the United States and Japan [21, 22]. In Western countries, the behaviour of drivers is controlled by traffic rules. Lajunen and Parker [23] examined the feasibility of using the Driving Anger Scale (DAS) developed by Deffenbacher et al. [24], and a sample of British drivers revealed that the subscale of "police presence" did not evoke any appreciable levels of anger, indicating that UK drivers' attitudes to traffic enforcement are more positive than US drivers. As traffic conditions and driving behaviour are different in different countries, it is not appropriate to expect that psychological instruments which have been developed elsewhere could work directly for traffic studies in other countries $[25,26]$.

Driving styles and the relationships between the different aspects of driving styles are still poorly understood [27] largely due to traffic culture, sociodemographic characteristics, and cultural differences [28]. This study aims to explore the effectiveness of the MDSI in two different geographical regions by conducting an independent validation of the questionnaire. It first validates the eight main factors and loadings through the distribution of the same questionnaire among drivers in China and the United Kingdom, thereby providing valid information on culturally relevant driving styles and becoming a valuable tool with which to support future studies of traffic psychology. It then explores the relationship between the identified driving styles and the sociodemographic characteristics. It concludes with a summary of the main findings and some suggestions for future directions of driving style research. This study contributes to efforts to promote road safety through an understanding of driving styles. 


\section{Research Experiment}

2.1. Participants. A total of 455 drivers were sampled, with 215 from China and 240 from the United Kingdom, who completed the questionnaire on which this study is based. The two groups were different in terms of gender, experience, and age distribution. The sample from diverse geographical areas in China consisted of 137 men and 78 women, who ranged in age (from 18 to 65 ) and in driving experience (consisting of the number of years of driving experiences from 1-40 years). The sample from the United Kingdom consisted of 127 men and 113 women, who ranged in age from 20 to 76 and had driving experience ranging from 2 to 58 years. The samples were divided into three groups according to age ("young" = younger than 30 years old, "Middle age" = between 31 and 60 years old, and "old" $=61$ years and older). The descriptive statistics for participant demographics are shown in Table 1.

It is expected that the demographic distribution of our sample reflects some degree of distribution within the respective driver population. For instance, a recent population-based controlled study in a capital city in China indicated a proportion of $18.5 \%$ young drivers, $61 \%$ of middle-aged drivers, and $20 \%$ older drivers [29]. In contrast, the age distribution of drivers in the United Kingdom is $27.5 \%$ for the under-30 group, $60 \%$ for the $31-60$ group, and $12.5 \%$ for the group aged above 61 [30]. Another study investigating the influence of gender on advanced driverassistance systems under Chinese road conditions had 50\% more male drivers than female drivers [31]. While the sample demographics seem reasonable, there is a lack of official information on the overall driver population distribution in this context to confirm the complete appropriateness of our sample selection [32].

2.2. Instruments. Taubman-Ben-Ari et al. [7] summarized the various developed measures of driving behaviour into a multidimensional conceptualization of driving styles. They broadened the scope of analysis from the behaviours related to traffic accidents, to behaviours, and habits related to daily driving. The original items in the MDSI were adapted primarily from several existing surveys, including the Driver Style Questionnaire (DSQ, French et al. [33]), the Driver Behaviour Questionnaire (DBQ, Reason et al. [34]), and the Driver Behaviour Inventory (DBI, Gulian et al. [35]). Each questionnaire taps very different aspects of driving styles. For instance, the DSQ [33] assesses factors such as "speed," "seat belt use," and "traffic violations," but also "feelings of control" and "risk-taking behaviour." In contrast, the DBI [35] studies "driving stress" and "dimensions of driving aggression," such as frustration relating to overtaking manoeuvres. The well-known DBQ [34] has yet another focus; it examines "lapses" (e.g., switching on the wrong appliance), "errors" (e.g., failing to notice pedestrians crossing the road), and deliberate "violations" (e.g., speeding or tailgating). Taubman-Ben-Ari et al. [7, 36] integrated the various measurement scales into a multidimensional conceptualization of driving styles and eventually formed the original version of the MDSI. This original MDSI, consisting of 44 items was created based on eight factors and four domains of driving styles: (1) a reckless and careless driving style, (2) an anxious driving style, (3) an angry and hostile driving style, and (4) a patient and careful driving style. The reckless and careless driving style refers to deliberate violations of safe driving norms, and the seeking of sensations and thrills while driving. It is characterized by driving at high speeds, passing in no-passing zones, and driving while intoxicated. The anxious driving style reflects feelings of alertness and tension, as well as ineffective engagement in relaxing activities during driving. The angry and hostile driving style refers to expressions of irritation, rage, and hostility while driving, along with a tendency to act aggressively on the road, including cursing other drivers, honking the horn, or flashing headlights. The patient and careful driving style reflects well-adjusted driving behaviours, such as planning, paying full attention to the road, displaying patience, courtesy, and calmness behind the wheel and obeying the traffic rules.

The Chinese version of the MDSI was developed based on the original MDSI [7]. The translation was conducted independently by two researchers and was crossed checked by a professional translator. The final version consisted of 44 items based on eight driving style factors that previous instruments used to measure as separate concepts (e.g., driving stress, driver aggression, and risky driving). A pilot test was conducted with six Chinese drivers to check the translated MDSI. All participants indicated that the Chinese version of the MDSI was understandable. Participants were asked to rate each item on a 6-point Likert-type scale (ranging from "not at all" to "very much") according to their behaviours during driving. It also incorporates positive driving styles, or more specifically, the patient and careful driving styles, which had been omitted from most previous studies (e.g., [37]).

2.3. Procedure. Participants were first recruited online via social media, having passed through screening procedures to ensure they qualified for the study. All participants were required to satisfy the following conditions: (1) at least 18 years old; (2) having a valid driving license; and (3) having at least 1 year of driving experience. The questionnaire was distributed with the online survey tool Sojump (http://www. sojump.com) through snowball sampling, in which the questionnaires were first disseminated to a group of university students, who were then asked to share the questionnaires with their network. Sojump is a professional online survey platform that permits services of questionnaire design and data collection. The recruited participants were presented with a brief overview of the purpose of the research and the eligibility criteria, and they were asked questions concerning their sociodemographic characteristics as well as their driving styles and driving behaviours under various passenger conditions. The two groups completed the survey in their respective mother tongue through the online survey tool. It lasted for a period of 3 months for the data collection and 1 month for monitoring the data 
TABle 1: Participants' demographics.

\begin{tabular}{lcc}
\hline Demographic variables & China sample & UK sample \\
\hline Gender & 137 men and 78 women & 127 men and 113 women \\
Age & Mean $=37.93, \mathrm{SD}=10.76$ & Mean $=49.14, \mathrm{SD}=14.069$ \\
$<30$ & 69 & 24 \\
$31-60$ & 141 & 156 \\
$>60$ & 5 & 60 \\
Driving years & Mean $=9.06, \mathrm{SD}=6.785$ & $\mathrm{Mean}=27.46, \mathrm{SD}=15.019$ \\
\hline
\end{tabular}

acquired. Ethical approval for the project was granted by the Research Ethics Subcommittee of the University of Nottingham, Ningbo.

2.4. Data Analysis. A Kolmogorov-Smirnov test was firstly conducted, which showed that the sample data were normally distributed $(P<0.05)$. A factor analysis was then performed to validate the previously developed MDSI versions with our samples. The mean substitution was used for the treatment of missing data. The factor analysis performed employed a Varimax rotation, to reveal the underlying structure of the items. The rotation method Oblimin was employed, which allows for the factors in the final solution to be correlated. We examined differences in these factor scores through sociodemographic and drivinghistory variables, employing multivariate analyses of covariance (MANCOVA). The univariate effects of the variables that emerged as significantly associated with factor scores were then further examined through univariate ANOVAs and Pearson's correlations.

\section{Results}

3.1. Factor Analysis of the Chinese Sample. A factor analysis with the Varimax rotation of the answers from the 215 Chinese participants was conducted to explore whether the 44 MDSI items would form the distinguishable eight factors as presumed when compared to the results of Taubman-BenAri et al. [7]. The findings reveal a distribution of the 44 items over eight factors (eigenvalue $>1$ ), explaining $56.1 \%$ of the variance. Two factors did not have sufficient items to make a meaningful contribution. Table 2 presents the loadings of each item.

Factor 1 consists of 11 items explaining $10.56 \%$ of the variance (Cronbach's alpha: .897). Four items in this factor were included in the anxious driving style, six in the dissociative driving style, and one in the high-velocity driving style of the original MDSI. Consequently, this factor was labelled as the angry and dissociative driving style. Factor 2 consists of seven items addressing risky, hostile, and thrillseeking behaviour. This factor explains $4.33 \%$ of the variance (Cronbach's alpha .886). Among these items, three were included in the risky driving style while three were in the angry driving style and one in the careful driving style of the original MDSI. This factor was labelled as the risky and angry driving style. Factor 3 consists of eight items addressing a person's tendency to drive quickly and to be oriented towards high-velocity driving. This factor explains $3.05 \%$ of the variance (Cronbach's alpha: .705). Five of these items were included in the high-velocity driving style, two in the anxious driving style, and one in the dissociative driving style of the original MDSI, which was labelled the highvelocity driving style. Factor 4 consists of six items addressing a well-adjusted and adaptive driving style, including concentration, patience, and compliance with road traffic regulations. This factor explains $2.08 \%$ of the variance (Cronbach's alpha: .705) and was labelled the patient and careful driving style. Three items of this factor were included in the patient driving style: two were in the careful driving style and one in the dissociative driving style of the original MDSI. Factor 5 consists of six items addressing a person's tendency to engage in relaxing activities to reduce stress while driving. This factor explains $1.38 \%$ of the variance (Cronbach's alpha: .73), among which four items were included in the distressed driving style, two in the careful, and one in the patient driving styles of the original MDSI. This factor was labelled as the distress-reduction driving style. Factor 6 consists of four items addressing inattentive driving behaviours when driving, which can be observed as an instantaneous attention deviation from driving. This factor was labelled as the inattentive driving style. This factor explains $1.38 \%$ of the variance (Cronbach's alpha: .83). There were two items from the dissociative driving style: one item from the distress-reduction driving style and one from the careful driving style of the original MDSI. Factor 7 consists of two items from the anxious driving behaviour of the original MDSI addressing confident driving behaviour. This factor explains $1.05 \%$ of the variance (Cronbach's alpha: .584 ), which was labelled as confident driving behaviour. Factor 8 consists of two items that explain $1.02 \%$ of the variance (Cronbach's alpha: .11), among which one item was included in the anxious driving behaviour and one in the high-velocity driving behaviour of the original MDSI. This factor was labelled as others.

A constraint was set to have at least three items per factor. The reliability of these factors was tested by Cronbach's alpha. Nunnally [41] stipulated that if the constructs are generally above or close to 0.70 , then it can be confirmed that the item measurements of the constructs are reliable. The items for Factor 7 achieved a low Cronbach's alpha figure, so it was removed from the analysis. The items in Factor 8 are heterogeneous, which makes it difficult to label, in combination with a low Cronbach's alpha, so this factor was also removed from the analysis.

As a follow-up to the results of the first-factor analysis, a second-factor analysis with a Varimax rotation consisting of six factors was conducted, in which items 4, 40, 2, and 7 were 
TABLE 2: Factor model coefficients of the multidimensional driving styles of the Chinese sample.

\begin{tabular}{|c|c|}
\hline Factors and items & Loading \\
\hline \multicolumn{2}{|l|}{ Factor 1: anxious and dissociated driving style } \\
\hline [30] Feel nervous while driving & 0.771 \\
\hline [35] Plan my route badly, so that I hit traffic that I could have avoided & 0.759 \\
\hline [37] Nearly hit something due to misjudging my gap in a parking lot & 0.743 \\
\hline [33] Intend to switch on the windscreen wipers but switch on the lights instead & 0.719 \\
\hline [32] Feel distressed while driving & 0.707 \\
\hline [29] Misjudge the speed of an oncoming vehicle when passing & 0.663 \\
\hline [26] Forget that my lights are on the full-beam until flashed by another motorist & 0.647 \\
\hline [9] Driving makes me feel frustrated & 0.635 \\
\hline [19] Attend to my hair/makeup while driving & 0.546 \\
\hline [24] It worries me when driving in bad weather & 0.522 \\
\hline [31] Get impatient during the rush hour & 0.511 \\
\hline \multicolumn{2}{|l|}{ Factor 2: risky and angry driving style } \\
\hline [23] Like the thrill of flirting with death or disaster & 0.826 \\
\hline [28] Get a thrill out of breaking the law & 0.774 \\
\hline [38] Enjoy the excitement of dangerous driving & 0.734 \\
\hline [21] Like to take risks while driving & 0.731 \\
\hline [4] Enjoy the sensation of driving at the limit & 0.607 \\
\hline [39] Honk my horn at others & 0.594 \\
\hline [27] When someone does something on the road that annoys me, I flash them with the high beam & 0.579 \\
\hline [11] Swear at other drivers & 0.30 \\
\hline \multicolumn{2}{|l|}{ Factor 3: high-velocity driving style } \\
\hline [15] In a traffic jam, I think about ways to get through the traffic faster & 0.691 \\
\hline [8] When in a traffic jam and the lane next to me starts to move, I try to move into that lane as soon as possible & 0.686 \\
\hline [3] Drive through traffic lights that have just turned red & 0.673 \\
\hline $\begin{array}{l}\text { [16] When a traffic light turns green and the car in front of me does not get going immediately, I try to urge the driver to move } \\
\text { on }\end{array}$ & 0.665 \\
\hline [1] Blow my horn or "flash" the car in front as a way of expressing frustration & 0.657 \\
\hline [18] When someone tries to skirt in front of me on the road, I drive in an assertive way in order to prevent it & 0.651 \\
\hline [34] Attempt to drive away from traffic lights in third gear (or in the neutral mode in automatic cars) & 0.468 \\
\hline \multicolumn{2}{|l|}{ Factor 4: patient and careful driving style } \\
\hline [17] At an intersection where I have to give right-of-way to oncoming traffic, I wait patiently for cross-traffic to pass & 0.762 \\
\hline [12] When a traffic light turns green and the car in front of me does not get going, I just wait for a while until it moves & 0.743 \\
\hline [40] Tend to drive cautiously & 0.655 \\
\hline [22] Base my behaviour on the motto "better safe than sorry" & 0.606 \\
\hline [13] Drive cautiously & 0.330 \\
\hline \multicolumn{2}{|l|}{ Factor 5: distress-reduction driving style } \\
\hline [36] Use muscle relaxation techniques while driving & 0.729 \\
\hline [41] Plan long journeys in advance & 0.659 \\
\hline [42] Always ready to react to unexpected manoeuvres by other drivers & 0.618 \\
\hline [7] While driving, I try to relax myself & 0.507 \\
\hline [6] Do relaxing activities while driving & 0.30 \\
\hline
\end{tabular}

Factor 6: inattentive driving style

[20] Distracted or preoccupied, and suddenly realize the vehicle ahead has slowed down, and have to slam on the brakes to avoid a collision

[14] Lost in thoughts or distracted, I fail to notice people at pedestrian crossings 0.579

[10] I daydream to pass the time while driving 0.546

[25] Meditate while driving 0.466

Factor 7

[2] Feel I have control over driving [-]

[43] Feel comfortable while driving

Factor 8

[44] Purposely tailgate other drivers

[5] On a clear freeway, I usually drive at or a little below the speed limit

The grey items were removed from the second analysis; the items in italics do not correlate with the same items in the original factor analysis.

excluded as they did not fit in the six main factors used in this analysis. The analysis revealed a distribution of 40 items over six factors explaining $58 \%$ of the variance. The loadings of the items for each of these factors are presented in Table 2.
Factor 1 consists of 11 items explaining 26.36\% of the variance (Cronbach's alpha: .902) addressing anxious and dissociative driving behaviour. Among these items, four were included in the anxious driving style, six in the 
dissociative driving style, and one in the high-velocity driving style of the original MDSI, which was labelled as the anxious and dissociative driving style. Factor 2 consists of eight items explaining $10.9 \%$ of the variance (Cronbach's alpha: .886). Four of these items were included in risky driving style: three in the angry driving style and one in the careful driving style of the original MDSI. This factor was labelled as the risky and angry driving style. Factor 3 consists of seven items explaining $7.58 \%$ of the variance (Cronbach's alpha: .76), among which five items were included in the high-velocity driving style, one in the anxious driving style, and one in the dissociative driving style of the original MDSI. This factor was labelled as the high-velocity driving style. Factor 4 consists of five items explaining $4.8 \%$ of the variance (Cronbach's alpha: .68). Three items were included in the patient driving style and two in the careful driving style of the original MDSI. Therefore, this factor was labelled the patient and careful driving style. Factor 5 consists of five items explaining $3.34 \%$ of the variance (Cronbach's alpha: $0.63)$, among which three items were included in the distress-reduction driving style, one in the careful and one in the patient driving style of the original MDSI. This factor was labelled the distress-reduction driving style. Factor 6 consists of four items explaining 3.07\% of the variance (Cronbach's alpha: 0.71 . This is a new factor including two items from the dissociative driving style: one item from the careful driving style and one from the distress-reduction driving style, which was labelled as the inattentive driving style.

3.2. Driving Styles and Sociodemographic Factors of the Chinese Sample. The relationship between the identified driving styles and the sociodemographic characteristics of gender, age, and driving experience were investigated by multivariate and univariate analyses of variance (ANOVA). The multivariate ANOVA indicated a significant gender difference $F(6,215)=1.257, P<0.01$. Univariate ANOVAs showed that gender differences were not significant in the anxious and dissociative driving style $(1,215)=0.516$, $P>0.05$ (men mean $=2.53, \mathrm{SD}=1.04$; women mean $=2.43$, $\mathrm{SD}=0.94)$, the distress-reduction driving style $(1,215)=$ $2.78, P>0.05$ (men mean $=4.14, \mathrm{SD}=0.93$; women mean$=3.94, \mathrm{SD}=0.98)$, the inattentive driving style $(1,215)=$ $0.912, \quad P>0.05 \quad$ (men mean $=3.04, \quad \mathrm{SD}=0.98$; women mean $=3.02, S D=0.87$ ), but they indicated significant differences in the risky and angry driving style $F(1,215)=4.51$, $P<0.05$ (men mean $=2.27, \mathrm{SD}=0.08$; women mean $=2$, $\mathrm{SD}=0.72)$, in the patient and careful driving style $F(1$, $215)=4.93, P<0.05$ (men mean $=4.12, \mathrm{SD}=0.90$; women mean $=4.37, \mathrm{SD}=0.76)$, and in the high-velocity driving style $F(1,215)=8.203, P<0.05$ (men mean $=3.63, \mathrm{SD}=1.13$; women mean $=3.1, \mathrm{SD}=1.03$ ).

Pearson's correlations ( ${ }^{1}$ Pearson's correlation coefficients between .10 and .29 represent a small association, coefficients between 0.30 and 0.49 represent a medium association, and coefficients of 0.50 and above represent a large association [45].) were tested to explore the associations between age and the six driving styles. They indicated that age was inversely associated with the anxious and dissociative driving style, $r(207)=-0.312, P<0.05$, the risky and angry driving style, $r(207)=-0.346, P<0.05$, the highvelocity driving style, $r(207)=-0.238, P<0.05$, distressreduction driving style, $r(207)=-0.205, P<0.05$, and the inattentive driving style, $r(207)=-0.314, P<0.05$ and that it was positively associated with the patient and careful driving style, $r(207)=.236, P<0.05$. This confirms that the older the participants, the lower the tendency to adopt anxious and dissociative, risky and angry, high-velocity, distress-reduction, and distracted driving styles, and the higher the tendency to embrace the patient and careful driving style.

Pearson's correlation also showed that driving experience was significantly inversely associated with the anxious and dissociative driving style, $r(207)=-0.237, P<0.05$, the risky and angry driving style, $r(215)=-0.241, P<0.05$, and the inattentive driving style, $r(207)=-0.313, P<0.05$, while it was positively associated with the patient and careful driving style, $r(207)=0.287, P<0.05$. It revealed no significant relationships between age and the high-velocity style and the distress-reduction driving style.

3.3. Factor Analysis of the UK Sample. The factor analysis with the Varimax rotation was conducted on the answers from the 240 UK participants, revealing distribution of the 44 items over eight factors explaining $65.7 \%$ of the variance. Two factors did not have sufficient items to make a meaningful contribution. The item distribution can be found in Table 3.

Factor 1 consists of five items explaining $34.1 \%$ of the total variance (Cronbach's alpha: .502). These items were labelled as the anxious driving style, explaining a person's tendency to experience nervousness and stress while driving. Factor 2 includes 19 items explaining $8.48 \%$ of the total variance (Cronbach's alpha: .962). These items refer to a person's tendency to seek stimulation and engagement in risky driving and the tendency to be distracted during driving, which was labelled as the risky and dissociative driving styles. Among these items, four items are included in the risky driving style, and seven in the dissociative driving style of the original MDSI, while the remaining eight items were from the other styles of the original MDSI. Factor 3 includes five items explaining $7.3 \%$ of the total variance (Cronbach's alpha: .804). This factor describes a person's tendency to drive quickly, and this kind of hazardous driving behaviour, which may interfere with and endanger other road users, was labelled as the high-velocity and angry driving style. Three items are included in the high-velocity driving style and two in the angry style of the original MDSI. Factor 4 includes five items explaining 3.21\% of the total variance (Cronbach's alpha: .623), describing people's tendency to be polite to other road users and exhibiting no sign of pressure on their time. Three items were previously included in the patient style in the MDSI, and two items were included in other driving styles. This factor was labelled as the patient driving style. Factor 5 includes three items explaining $2.09 \%$ of the total variance (Cronbach's alpha: .784), in which people tend to drive carefully and in a structured manner. This factor was labelled as the careful 
TABLE 3: Factor model coefficients of the multidimensional driving styles of the UK sample.

\begin{tabular}{|c|c|}
\hline Factors and items & Loading̨ \\
\hline \multicolumn{2}{|l|}{ Factor 1: anxious driving style } \\
\hline [43] Feel comfortable while driving & 0.753 \\
\hline [30] Feel nervous while driving & 0.728 \\
\hline [24] It worries me when driving in bad weather & 0.674 \\
\hline [2] Feel I have control over driving [-] & 0.580 \\
\hline [9] Driving makes me feel frustrated & 0.571 \\
\hline [32] Feel distressed while driving & 0.569 \\
\hline \multicolumn{2}{|l|}{ Factor 2: risky and dissociative driving style } \\
\hline [23] Like the thrill of flirting with death or disaster & 0.857 \\
\hline [19] Attend to my hair/makeup while driving & 0.835 \\
\hline [14] Lost in thoughts or distracted, I fail to notice people at pedestrian crossings & 0.808 \\
\hline $\begin{array}{l}\text { [20] Distracted or preoccupied and suddenly realize the vehicle ahead has slowed down and have to slam on the brakes to avoid a } \\
\text { collision }\end{array}$ & 0.802 \\
\hline [34] Attempt to drive away from traffic lights in third gear (or in the neutral mode in automatic cars) & 0.794 \\
\hline [29] Misjudge the speed of an oncoming vehicle when passing & 0.788 \\
\hline [28] Get a thrill out of breaking the law & 0.787 \\
\hline [21] Like to take risks while driving & 0.782 \\
\hline [26] Forget that my lights are on the full-beam until flashed by another motorist & 0.777 \\
\hline [3] Drive through traffic lights that have just turned red & 0.777 \\
\hline [38] Enjoy the excitement of dangerous driving & 0.762 \\
\hline [44] Purposely tailgate other drivers & 0.739 \\
\hline [37] Nearly hit something due to misjudging my gap in a parking lot & 0.734 \\
\hline [33] Intend to switch on the windscreen wipers but switch on the lights instead & 0.689 \\
\hline [35] Plan my route badly, so that I hit traffic that I could have avoided & 0.642 \\
\hline [27] When someone does something on the road that annoys me, I flash them with the high beam & 0.519 \\
\hline [25] Meditate while driving & 0.457 \\
\hline \multicolumn{2}{|l|}{ Factor 3: high-velocity and angry driving style } \\
\hline [15] In a traffic jam, I think about ways to get through the traffic faster & 0.708 \\
\hline [31] Get impatient during the rush hour & 0.699 \\
\hline $\begin{array}{l}\text { [16] When a traffic light turns green and the car in front of me does not get going immediately, I try to urge the driver to move } \\
\text { on }\end{array}$ & 0.626 \\
\hline [1] Blow my horn or "flash" the car in front as a way of expressing frustration & 0.623 \\
\hline [18] When someone tries to skirt in front of me on the road, I drive in an assertive way in order to prevent it & 0.607 \\
\hline [39] Honk my horn at others & 0.508 \\
\hline [8] When in a traffic jam and the lane next to me starts to move, I try to move into that lane as soon as possible & 0.455 \\
\hline \multicolumn{2}{|l|}{ Factor 4: patient driving style } \\
\hline [12] When a traffic light turns green and the car in front of me does not get going, I just wait for a while until it moves & 0.714 \\
\hline [17] At an intersection where I have to give right-of-way to oncoming traffic, I wait patiently for cross-traffic to pass & 0.610 \\
\hline [41] Plan long journeys in advance & 0.591 \\
\hline [42] Always ready to react to unexpected manoeuvres by other drivers & 0.440 \\
\hline \multicolumn{2}{|l|}{ Factor 5: careful driving style } \\
\hline [40] Tend to drive cautiously & 0.784 \\
\hline [13] Drive cautiously & 0.756 \\
\hline [22] Base my behaviour on the motto "better safe than sorry" & 0.585 \\
\hline \multicolumn{2}{|l|}{ Factor 6: distress-reduction driving style } \\
\hline [6] Do relaxing activities while driving & 0.768 \\
\hline [7] While driving, I try to relax myself & 0.724 \\
\hline [36] Use muscle relaxation techniques while driving & 0.655 \\
\hline
\end{tabular}

\section{Factor 7}

[4] Enjoy the sensation of driving on the limit

[5] On a clear freeway, I usually drive at or a little below the speed limit

Factor 8

[11] Swear at other drivers

[10] I daydream to pass the time while driving

The grey items were removed from the second analysis; the items in italics do not correlate with the same items in the original factor analysis.

driving style. Two items were included in the careful style and the remaining item in the patient style of the original MDSI. Factor 6 includes three items explaining 1.5\% of the total variance (Cronbach's alpha: .562), describing drivers' engagement in relaxing activities while driving, which was labelled as the distress-reduction driving style. These three items were included in the original MDSI, corresponding to the distress-reduction style. Factor 7 includes two items 
explaining $1.1 \%$ of the total variance (Cronbach's alpha: .201). These items were part of the risky and anxious driving styles of the original MDSI. Factor 8 includes two items explaining $1.02 \%$ of the total variance, and these were included in the dissociative driving style of the original MDSI.

A constraint was again set to have at least three items per factor. The items in Factors 7 and 8 achieved a low Cronbach's alpha figure and were removed from the analysis. As a follow-up to the results of the first-factor analysis, a secondfactor analysis with the Varimax rotation consisting of six factors was conducted, in which items 6, 7, and 12 were excluded. This analysis revealed a distribution of 41 items over six factors, explaining $62.86 \%$ of the variance. The loadings of the items on each of these factors are presented in Table 3.

Factor 1 includes six items explaining $35.43 \%$ of the total variance (Cronbach's alpha: .585). All items were included in the anxious driving style of the original MDSI. Factor 2 includes 17 items explaining $8.81 \%$ of the total variance (Cronbach's alpha: .960). These items address unsafe and risky driving behaviour, among which four items were included in the risky and dissociative driving styles, seven in the dissociative driving style of the original MDSI, and the remaining six items from other styles in the original MDSI. Factor 3 includes seven items explaining $7.4 \%$ of the total variance (Cronbach's alpha: .831). Four items are included in the high-velocity driving style, and three in the angry style of the original MDSI. Factor 4 includes four items addressing patient driving behaviour and attitudes. This factor explains $4.91 \%$ of the total variance (Cronbach's alpha: .623). Among these items, three were previously included in the patient style and one in the careful driving style of the original MDSI. Factor 5 includes three items explaining 3.48\% of the total variance (Cronbach's alpha: .784). There are two items in the careful style and one in the patient style of the original MDSI. Factor 6 consists of three items explaining $2.83 \%$ of the total variance (Cronbach's alpha: .562), and this was labelled the distress-reduction driving style.

3.4. Driving Styles and Sociodemographic Factors of the UK Sample. The relationship between the identified driving styles and the sociodemographic characteristics of gender, age, and driving experience were investigated by multivariate and univariate analyses of variance (ANOVA). The multivariate ANOVA indicated no significant gender difference $F(6,240)=2-16, P>0.05$. Univariate ANOVAs showed that gender differences were significant in the risky and dissociative driving styles $F(1,240)=0.9 .33, P<0.05$, (men mean $=1.92, \quad \mathrm{SD}=1.06$; women mean $=1.57$, $\mathrm{SD}=0.63)$ and not significant in the anxious driving style $F$ $(1,240)=0.394, \quad P>0.05 \quad($ men mean $=3.36, \quad \mathrm{SD}=.71$; women mean $=3.41, \mathrm{SD}=0.57)$, the high-velocity and angry driving style $F(1,240)=0.3 .07, P>0.05$ (men mean $=2.79, \mathrm{SD}=1.06$; women mean $=2.55, \mathrm{SD}=1.01)$, the patient driving style $F(1,240)=0.216, P>0.05$ (men mean $=4.54, \mathrm{SD}=0.91$; women mean $=4.71, \mathrm{SD}=0.84)$, the careful driving style $F(1,240)=0.225, P>0.05$ (men mean $=4.59, \mathrm{SD}=0.97$; women mean $=4.78, \mathrm{SD}=1.01$ ), and the distress-reduction driving style $F(1,240)=0.764$, $P>0.05$ (men mean $=4.59, \mathrm{SD}=0.97$; women mean $=4.78$, $\mathrm{SD}=1.01)$.

Pearson's correlations indicated inverse associations between age and the anxious driving style, $r(235)=-0.118$, $P<0.05$, the risky and dissociative driving style, $r(235)=-$ $0.401, P<0.01$, the high-velocity and angry driving style, $r$ (235) $=-0.481, P<0.05$, and the distress-reduction driving style, $r(235)=-0.315, P<0.01$. There was no significant relationship identified between age and the patient driving style, $r(235)=0.06, P>0.05$, or age and the careful driving style, $r(235)=0.09, P>0.05$. This provides evidence that the older the participant, the lower the tendency to adopt anxious, risky, high-velocity, and distress-reduction driving styles.

Pearson's correlation also showed that driving experience was inversely associated with the anxious driving style, $r(235)=-0.215, P<0.05$, the risky and dissociative driving style, $r(235)=-0.493, P<0.01$, the high-velocity and angry driving style, $r(235)=-0.419, P<0.05$, and the distressreduction driving style, $r(235)=-0.328, P<0.01$. It revealed no significant relationships between age and the careful driving style, $r(235)=0.32, P>0.05$, and between age and the patient driving style, $r(235)=0.22, P>0.05$.

\section{Conclusion and Discussion}

The analysis of the Chinese participants' dataset showed its factorial structure is comparable to the previous MDSI, with 40 of the 44 original items divided over six factors. Two of the eight factors were identical to the factorial composition of the MDSI. Six factors in the original MDSI [7] were merged into three groups. Specifically, the "anxious and dissociative", the "risky and angry," and the "patient and careful" driving styles form single dimensions in the MDSI.

The present findings also suggest the inattentive style as a new dimension in the Chinese sample. The "inattentive" driving style refers to inattentiveness to driving procedures, which is caused by cognitive distraction. Inattention while driving can be understood as an instantaneous cognitive deviation from normal behaviour with the immediate following of sudden driver actions to correct behaviours resulting from inattention [37]. An inattentive driving style differs from a dissociative driving style owing to its cognitive nature, as opposed to a dissociative driving style which can be observed as the practice of driving while engaging in another physical activity. Inattention plays a significant role in the field of road safety problems [43], and it was not given enough attention when Taubman-Ben-Ari et al. [7, 36] proposed the central domains of driving styles. According to an empirical study [42], 78\% of the 82 accidents recorded and $65 \%$ of the 761 near accidents were the direct result of driver inattention. Tango and Botta [40] estimated that $13.3 \%$ of accidents can be attributed to driver cognitive distraction, while $9.7 \%$ of crashes are categorized as incidents when the driver "looked but did not see." For example, with the pervasive use of mobile devices, the attention distraction caused by cell phone conversations has now become a common phenomenon [39]. 
Developing countries especially struggle to address road and vehicle safety concerns owing to an increased number of inattentive and careless drivers [37]. For example, the Ministry of Works of Malaysia has provided evidence that $41 \%$ of vehicle collisions are the direct result of driver carelessness [38]. The International Transport Forum's annual road safety report ranked South Africa as the country with the highest number of road fatalities [46], while there are studies of Chinese drivers' behaviour showing that inattentive driving made a significant contribution to traffic accidents (e.g., [47]). The complicated driving environment in China poses further challenges to these groups of inattentive drivers, including infrastructural issues, such as roads being constructed incorrectly [48], poorly designed, or a lack of road signs [49], and drivers having to share the road with a substantially large number of pedestrians and cyclists [50]. Uncertainty about the reactions and trajectories of vulnerable road users, such as those who are attempting to violate traffic control signals to cross at their own convenience, may further exacerbate the complexity of existing traffic situations [50]. It is evident that inattentive driving behaviour is considered a major safety problem, and there is significant benefit in identifying those drivers who engage in an inattentive driving style.

The results concerning the gender differences in the Chinese sample are consistent with previous literature, with men being more likely to engage in the risky and angry, and the high-velocity driving styles, while women are more likely to engage in patient and careful driving styles [51]. One possible explanation for this, as stated by Özkan and Lajunen [52], is that most male drivers exhibit high levels of masculine characteristics (e.g., being adventurous and aggressive), tending to view reckless driving as a challenge rather than a threat while interpreting the behaviour of other drivers as hostile. In contrast, the majority of female drivers are perceived as possessing highly feminine traits (e.g., caring for other people) so that they may prefer to drive in a safe and careful manner. There is no gender difference for the anxious and dissociative, or distress-reduction and inattentive driving styles, contrasting with Taubman-Ben-Ari and Skvirsky's study [36], which found female drivers scoring higher for the anxious style. The associations between age, driving experience, and the six driving styles were in line with previous studies, whereby age was inversely related to the maladaptive driving styles, namely anxious and dissociative, risky and angry, high-velocity, and inattentive [27] but also to the distress-reduction style, as in Poó et al. [8], while being positively related to the adaptive driving style, namely, patient and careful. This finding is also evidence of the influence of driving experience on driving styles, indicating that less-skilled drivers are more inclined to adopt anxious and dissociative, risky and angry, and inattentive driving styles [13]. It revealed no significant relationships between driving experience and the high-velocity and distress-reduction driving styles.

The analysis of the UK sample showed that most of the original factors could be replicated quite well, with 40 of the 44 original items divided over six factors. Four of the eight factors are identical to the factorial structure of the MDSI.
Two factors in the original MDSI were merged into one group, namely the high-velocity and angry driving style. The findings also indicated that the risky driving style of the original MDSI was combined with the dimension of the dissociative driving style, which formed a new group. The gender difference was found to be consistent with Özkan and Lajunen's study [52], indicating that men are more likely to adopt risky and dissociative driving styles. The findings further revealed the association between age, driving experience, and driving style, indicating that young and lessskilled drivers are more inclined to adopt anxious, risky and dissociative, high-velocity, and angry driving styles, which is consistent with some previous investigations $[4,13,27,36]$. However, there seems to be no clear relationship between age and experience and their effect on the adoption of careful and patient driving styles. This means that careful and patient styles may potentially be found among drivers of all ages and years of experience. A report has revealed that $91 \%$ of UK drivers self-report that they are "careful and competent" drivers [53]. Drivers who drive carefully and act patiently, even when traffic conditions do not satisfy their expectations, emphasize safety on the roads. The International Traffic Safety Data and Analysis Group accident database evaluated 37 countries and ranked the United Kingdom as one of the countries with the lowest number of road fatalities, with a ratio of 4 deaths per 100,000 inhabitants in 2011 [46].

Our findings imply that there is a need to explore and induce driver and road safety enhancement solutions through the investigation of driver styles. An increasing number of studies have employed psychological responses to collect data about drivers (e.g., $[9,11,14])$. Such responses in drivers could then be translated into adaptive and maladaptive driving styles. If such an assessment of driving styles could be identified, driver behaviour could potentially be improved by focusing education on shifting these responses from maladaptive to adaptive driving styles. For example, Ellison et al. [54] built a driver model to evaluate driver behaviour to improve road safety. This study also highlights how driving-related behaviours have been shown to vary across countries, including drivers' tendencies to obey or break traffic rules. As traffic conditions, living environments, and cultural backgrounds vary around the world, drivers exhibit different styles when participating in a traffic environment [13]. Any such differences also raise the need to adapt psychological responses to give consideration to cultural specificities $[18,19]$.

This study has some limitations. First, it relied on selfreported driving behaviour, and the results obtained from driving style questionnaires could be used as predictors of actual driving behaviour. However, there is a need to further investigate how the results of the self-reported behaviour are correlated with actual behaviour in a vehicle or driving simulator. This will validate the trustworthiness of the metric due to the reporting bias and will enhance the understanding of driving styles. Second, the study followed the guideline of Child [55]. Any factor loading scores less than 0.3 were removed. Scores greater than 0.4 were considered stable [56], which appeared in the British sample. In the Chinese sample, 
there were three items (i.e., one in Factor 2, one in Factor 4, and one in Factor 5), indicating that the factors account for approximately $30 \%$ of the relationship within the data. For small samples, 0.3 should be used with caution, although it was observed that for each of these factors, there were at least 3 non-cross-loading items with an acceptable loading score of 0.4 or above. Finally, both studies were conducted on convenience samples, which are not representative of the entire Chinese and UK populations, and while efforts were made to include drivers varying as much as possible in terms of their age and driving experience, some caution must be urged in generalizing the results.

\section{Data Availability}

The data are not publicly available due to restrictions, e.g., there contained information that could compromise the privacy of research participants. The data that support the findings of this study are available from the corresponding author upon reasonable request.

\section{Conflicts of Interest}

The authors declare that they have no conflicts of interest.

\section{References}

[1] F. Hartwich, M. Beggiato, and J. F. Krems, "Driving comfort, enjoyment and acceptance of automated driving-effects of drivers' age and driving style familiarity-'," Ergonomics, vol. 61, no. 8, pp. 1017-1032, 2018.

[2] T. Rothengatter and R. D. Huguenin, Traffic and Transport Psychology Theory and Application: Proceedings of the ICTTP 2000, Elsevier, Amsterdam, Netherland, 2004.

[3] J. Elander, R. West, and D. French, "Behavioral correlates of individual differences in road-traffic crash risk: an examination of methods and findings," Psychological Bulletin, vol. 113, no. 2, pp. 279-294, 1993.

[4] H. Gwyther and C. Holland, "The effect of age, gender and attitudes on self-regulation in driving," Accident Analysis \& Prevention, vol. 45, pp. 19-28, 2012.

[5] H. Hooft van Huysduynen, J. Terken, and B. Eggen, "The relation between self-reported driving style and driving behaviour. a," Transportation Research Part F: Traffic Psychology and Behaviour, vol. 56, pp. 245-255, 2018.

[6] A. H. Goodwin, R. D. Foss, and N. P. O'Brien, The Effect of Passengers on Teen Driver Behavior (No. DOT HS 811 540), National Highway Traffic Safety Administration, Washington, DC, USA, 2012.

[7] O. Taubman-Ben-Ari, M. Mikulincer, and O. Gillath, "The multidimensional driving style inventory-scale construct and validation," Accident Analysis \& Prevention, vol. 36, no. 3, pp. 323-332, 2004.

[8] F. M. Poó, O. Taubman-Ben-Ari, R. D. Ledesma, and C. M. Díaz-Lázaro, "Reliability and validity of a Spanishlanguage version of the multidimensional driving style inventory," Transportation Research Part F: Traffic Psychology and Behaviour, vol. 17, pp. 75-87, 2013.

[9] M. A. Trógolo, J. D. Tosi, F. M. Poó, R. D. Ledesma, L. A. Medrano, and S. Dominguez-Lara, "Factor structure and measurement invariance of the multidimensional driving style inventory across gender and age: an ESEM approach,"
Transportation Research Part F: Traffic Psychology and Behaviour, vol. 71, pp. 23-30, 2020.

[10] H. H. V. Huysduynen, J. Terken, J. Martens, and B. Eggen, "Measuring driving styles: a validation of the multidimensional driving style inventory," in Proceedings of the 7th International Conference on Automotive User Interfaces and Interactive Vehicular Applications, pp. 257-264, Nottingham, England, September 2015.

[11] J. L. Padilla, C. Castro, P. Doncel, and O. Taubman-Ben-Ari, "Adaptation of the multidimensional driving styles inventory for Spanish drivers: convergent and predictive validity evidence for detecting safe and unsafe driving styles," Accident Analysis \& Prevention, vol. 136, Article ID 105413, 2020.

[12] J. Karjanto, N. M. Yusof, J. Terken et al., “The identification of Malaysian driving styles using the multidimensional driving style inventory," in Proceedings of the 8th International Conference on Automotive User Interfaces and Interactive Vehicular Applications, pp. 245-252, Ann Arbor, MI, USA, October 2016.

[13] A. C. Holman and C. E. Havârneanu, "The Romanian version of the multidimensional driving style inventory: psychometric properties and cultural specificities," Transportation Research Part F: Traffic Psychology and Behaviour, vol. 35, pp. 45-59, 2015.

[14] F. Freuli, G. De Cet, M. Gastaldi et al., "Cross-cultural perspective of driving style in young adults: psychometric evaluation through the analysis of the multidimensional driving style inventory," Transportation Research Part F: Traffic Psychology and Behaviour, vol. 73, pp. 425-432, 2020.

[15] Y. Wang, W. Qu, Y. Ge, X. Sun, and K. Zhang, "Effect of personality traits on driving style: psychometric adaption of the multidimensional driving style inventory in a Chinese sample," PLoS One, vol. 13, no. 9, Article ID e0202126, 2018.

[16] S. Long and C. Ruosong, "Reliability and validity of the multidimensional driving style inventory in Chinese drivers," Traffic Injury Prevention, vol. 20, no. 2, pp. 152-157, 2019.

[17] M. Houtenbos, "Expecting the unexpected," Thesis, Delft University of Technology, Leidschendam, The Netherlands, 2008.

[18] D. Yang, X. Qiu, D. Yu, and R. Sun, "Study on the driving style heterogeneity and drivers' driving styles choice in China," in Proceedings of the Annual Meeting Transportation Research Board 01558168, Washington, DC, USA, January 2015.

[19] Z. Feng, S. Lu, and W. Zhang, "Difference and similarity comparisons of traffic accident characteristics in China and Europe," in Proceedings of the 14th COTA International Conference of Transportation Professionals, pp. 2666-2676, American Society of Civil Engineers, Changsha, China, 2014.

[20] F. Li, X. Yao, L. Jiang, and Y. Li, "Driving anger in China: psychometric properties of the driving anger scale (DAS) and its relationship with aggressive driving," Personality and Individual Differences, vol. 68, pp. 130-135, 2014.

[21] P. Atchley, J. Shi, and T. Yamamoto, "Cultural foundations of safety culture: a comparison of traffic safety culture in China, Japan and the United States," Transportation Research Part F: Traffic Psychology and Behaviour, vol. 26, pp. 317-325, 2014.

[22] W. Zhang, O. Tsimhoni, M. Sivak, and M. J. Flannagan, "Road safety in China: analysis of current challenges," Journal of Safety Research, vol. 41, no. 1, pp. 25-30, 2010.

[23] T. Lajunen and D. Parker, "Are aggressive people aggressive drivers? a study of the relationship between self-reported general aggressiveness, driver anger and aggressive driving," Accident Analysis \& Prevention, vol. 33, no. 2, pp. 243-255, 2001. 
[24] J. L. Deffenbacher, M. E. Huff, R. S. Lynch, E. R. Oetting, and N. F. Salvatore, "Characteristics and treatment of high-anger drivers," Journal of Counseling Psychology, vol. 47, no. 1, pp. 5-17, 2000.

[25] O. Şimşekoğlu, T. Nordfjærn, M. F. Zavareh, A. M. Hezaveh, A. R. Mamdoohi, and T. Rundmo, "Risk perceptions, fatalism and driver behaviors in Turkey and Iran," Safety Science, vol. 59, pp. 187-192, 2013.

[26] T. Nordfjærn and T. Rundmo, "Perceptions of traffic risk in an industrialized and a developing country," Transportation Research Part F, vol. 12, pp. 91-98, 2009.

[27] F. Sagberg, G. B. Selpi, G. F. Bianchi Piccinini, and J. Engström, "A review of research on driving styles and road safety," Human Factors: The Journal of the Human Factors and Ergonomics Society, vol. 57, no. 7, pp. 1248-1275, 2015.

[28] T. Özkan, T. Lajunen, J. E. Chliaoutakis, D. Parker, and H. Summala, "Cross-cultural differences in driving behaviours: a comparison of six countries," Transportation Research Part F: Traffic Psychology and Behaviour, vol. 9, no. 3, pp. 227-242, 2006.

[29] B. Shen, W. Qu, Y. Ge, X. Sun, and K. Zhang, "The relationship between personalities and self-report positive driving behavior in a Chinese sample," PloS One, vol. 13, Article ID e0190746, 2018.

[30] K. R. Thompson, A. M. Johnson, J. L. Emerson, J. D. Dawson, E. R. Boer, and M. Rizzo, "Distracted driving in elderly and middle-aged drivers," Accident Analysis \& Prevention, vol. 45, no. 2, pp. 711-717, 2012.

[31] G. Li, S. Eben Li, and B. Cheng, "Field operational test of advanced driver assistance systems in typical Chinese road conditions: the influence of driver gender, age and aggression," International Journal of Automotive Technology, vol. 16, no. 5, pp. 739-750, 2015.

[32] W. J. Stevenson, Operations Management, McGraw-Hill Education, New York, NY, USA, 12th edition, 2014.

[33] D. J. French, R. J. West, J. Elander, and J. M. Wilding, "Decision-making style, driving style, and self-reported involvement in road traffic accidents," Ergonomics, vol. 36, no. 6, pp. 627-644, 1993.

[34] J. T. Reason, A. Manstead, S. G. Stradling, J. Baxter, and K. Campbell, "Errors and violations on the road-a real distinction," Ergonomics, vol. 33, no. 11, pp. 1315-1332, 1990.

[35] E. Gulian, G. Matthews, A. I. Glendon, D. R. Davies, and L. M. Debney, "Dimensions of driver stress," Ergonomics, vol. 32, no. 6, pp. 585-602, 1989.

[36] O. Taubman-Ben-Ari and V. Skvirsky, "The multidimensional driving style inventory a decade later: review of the literature and re-evaluation of the scale," Accident Analysis \& Prevention, vol. 93, pp. 179-188, 2016.

[37] G. Albertus, M. Meiring, and H. C. Myburgh, "A review of intelligent driving style analysis systems and related artificial intelligence algorithms," Sensors, vol. 15, pp. 30653-30682, 2015.

[38] I. Mohamad, M. Ali, and M. Ismail, "Abnormal driving detection using real time global positioning system data," in Proceedings of the 2011 IEEE International Conference on Space Science and Communication (IconSpace), pp. 1-6, IEEE, Penang, Malaysia, July 2011.

[39] K. L. Young, C. M. Rudin-Brown, C. Patten, R. Ceci, and M. G. Lenné, "Effects of phone type on driving and eye glance behaviour while text-messaging," Safety Science, vol. 68, pp. 47-54, 2014.

[40] F. Tango and M. Botta, "Real-time detection system of driver distraction using machine learning," IEEE Transactions on
Intelligent Transportation Systems, vol. 14, no. 2, pp. 894-905, 2013.

[41] J. C. Nunnally, Psychometric Theory, McGraw-Hill, New York, NY, USA, 2nd edition, 1978.

[42] O. Bagdadi, "Assessing safety critical braking events in naturalistic driving studies," Transportation Research Part F: Traffic Psychology and Behaviour, vol. 16, pp. 117-126, 2013.

[43] V. L. Neale, T. A. Dingus, S. G. Klauer, J. Sudweeks, and M. Goodman, "An overview of the 100-car naturalistic study and findings," in Proceedings of the 19th International Technical Conference on the Enhanced Safety of Vehicles, pp. 6-9, ESV, Washington, DC, USA, June 2005.

[44] M. A. Regan and E. Mitsopoulos, "Understanding passenger influences on driver behaviour: implications for road safety and recommendations for countermeasure development," Report 180, Monash University Accident Research Centre, Melbourne, Australia, 2001.

[45] P. Bobko, Correlation and Regression: Applications for Industrial Organizational Psychology and Management, Sage Publications, Thousand Oaks, CA, USA, 2nd edition, 2001.

[46] International Traffic Safety Data and Analysis Group, International Traffic Safety Data and Analysis Group, 2020, http://www.internationaltransportforum.org/jtrc/safety/ safety.html Road Safety Annual Report.

[47] A. Lindgern, F. Chen, P. W. Jordan, and H. Zhang, "Requirements for the design of advanced driver assistance systems - the differences between Swedish and Chinese drivers," International Journal of Design, vol. 2, no. 2, pp. 41-54, 2008.

[48] J. Li, Z. Henk Van, and S. Lu, "Calibration of a traffic simulation for a Chinese city," in Proceedings of the 14th Meeting of the Euro Working Group on Transportation, Elsievier, Poznan, Poland, September 2011.

[49] Y.-H. Huang, W. Zhang, M. Roetting, and D. Melton, "Experiences from dual-country drivers: driving safely in China and the US," Safety Science, vol. 44, no. 9, pp. 785-795, 2006.

[50] P. Wang, P.-L. P. Rau, and G. Salvendy, "Road safety research in China: review and appraisal," Traffic Injury Prevention, vol. 11, no. 4, pp. 425-432, 2010.

[51] D. Shinar and R. Compton, "Aggressive driving: an observational study of driver, vehicle, and situational variables," Accident Analysis \& Prevention, vol. 36, no. 3, pp. 429-437, 2004.

[52] T. Özkan and T. Lajunen, "Why are there sex differences in risky driving? the relationship between sex and gender-role on aggressive driving, traffic offences, and accident involvement among young Turkish drivers," Aggressive Behavior, vol. 31, no. 6, pp. 547-558, 2005.

[53] 91\% of UK drivers say they're "careful and competent": Available at https://www.autoexpress.co.uk/car-news/ 105267/91-of-uk-drivers-say-theyre-careful-and-competent.

[54] A. B. Ellison, S. P. Greaves, and M. C. J. Bliemer, "Driver behaviour profiles for road safety analysis," Accident Analysis \& Prevention, vol. 76, pp. 118-132, 2015.

[55] D. Child, The Essentials of Factor Analysis, Bloomsbury Academic, London, UK, 3rd edition, 2006.

[56] E. Guadagnoli and W. F. Velicer, "Relation of sample size to the stability of component patterns," Psychological Bulletin, vol. 103, no. 2, pp. 265-275, 1988. 